\title{
Attenuation of ocular and systemic responses to tracheal intubation by intravenous lignocaine
}

\author{
BENJAMIN DRENGER' AND JACOB PE'ER'
}

From the Departments of 'Anaesthesiology and 'Ophthalmology, Hadassah University Hospital, Jerusalem, Israel

SUMMARY This study was undertaken to determine whether intravenous lignocaine could mitigate or prevent the ocular reactions and especially the acute increase in intraocular pressure associated with laryngoscopy and tracheal intubation. Two groups of children undergoing minor eye surgery under nitrous oxide-oxygen-halothane anaesthesia were examined. The experimental group $(\mathrm{n}=$ 17) received $2 \mathrm{mg} / \mathrm{kg}$ lignocaine and the controls $(n=18)$ an equivalent volume of saline. The incidence of local laryngeal and ocular reflexes was much lower in the lignocaine group. Pulse acceleration was significantly lower in the lignocaine group $(p<0.025)$, and the maximal mean intraocular pressure was significantly less than in the control group $(p<0.005)$. Other ocular reactions were recorded, and all were attenuated after lignocaine administration. The beneficial effects of lignocaine, a suppressant of autonomic reflexes, suggest that intraocular pressure, like the heart rate, rises after intubation as a result of autonomic stimulation. The use of intravenous lignocaine is thus recommended for children at risk, such as those needing an urgent operation because of lacerated eye injury under rapid sequence induction of anaesthesia.

Conspicuous systemic reactions accompany laryngoscopy and tracheal intubation. Previous studies have shown the ability of intravenous lignocaine to reduce these autonomic responses ${ }^{12}$ and its effectiveness as a cough suppressant. ${ }^{3}$

A brief elevation of intraocular pressure (IOP) during laryngoscopy and tracheal intubation is not harmful to the normal or even glaucomatous eye, but it may be hazardous to a lacerated and perforated globe or to the eye after recent surgery. ${ }^{+6}$ Intravenous lignocaine has been found to attenuate the rise in IOP caused by tracheal intubation. ${ }^{7}$ The purpose of the present study is to evaluate in children the overall .effects of intravenous lignocaine given prior to tracheal intubation on ocular, autonomic, and laryngeal responses.

\section{Materials and methods}

Thirty-five patients, aged 1-10 years, scheduled for minor eye surgery under general anaesthesia were included in the study. None of these children had a

Correspondence to J Pe'er, MD, Department of Ophthalmology, Hadassah University Hospital, PO Box 12000, 91120 Jerusalem, Isracl. history of glaucoma or intraocular disease, and they needed the operation for correction of strabismus or ptosis.

Pure inhalational anaesthetic technique was used, based on inhalation of mixture of nitrous oxide and oxygen with addition of halothane up to $2 \%$. Five minutes after induction of anaesthesia the initial measurements of heart rate, blood pressure, and IOP were taken, and pupillary reactions (miosis or mydriasis), lacrimation, and systemic reactions such as cough and breath holding were recorded by two observers by mutual agreement. Then an intravenous injection of $0.13 \mathrm{ml} / \mathrm{kg}$ of coded solution was given in a double blind randomised manner. The solution contained either $2 \mathrm{mg} / \mathrm{kg}$ lignocaine $\mathrm{HC} 1$ without preservative $(n=17)$ or normal saline $(n=18)$.

The intraocular pressure was measured with Schiøtz tonometer. The Schiøtz units were converted to $\mathrm{mmHg}$ by the appropriate calibration table for Schiøtz tonometry. Heart rate was recorded continuously by Mennen 741 electrocardiogram monitor and blood pressure was measured manually by sphygmomanometer.

The measured parameters were recorded 1 and 2 minutes after the injection of the coded solution. 
When the eyes were in midposition, pupils in miosis, and the mandible muscles well relaxed, tracheal intubation was performed using a non-cuffed tube and without addition of muscle relaxants. In all cases the intubation was smooth and uneventful. Immediately after intubation all measurements were repeated every minute for the next 4 minutes. Statistical analysis was performed by the Student's $t$ test.

\section{Results}

Seventeen children received intravenous injection of lignocaine and 18 children received saline. In the control saline group the intubation caused a significant acceleration in the heart rate from a mean of $93 \cdot 3$, SEM $3 \cdot 7$, beats per minute to $111 \cdot 7$, SEM $5 \cdot 3,(p<0 \cdot 01)$, while in the lignocaine group the change was non-significant, from $92 \cdot 6$, SEM $4 \cdot 6$, to $101 \cdot 6$, SEM $4 \cdot 7$, beats per minute $(p<0 \cdot 2)$.

The reactions of the patients to tracheal intubation are shown in Fig. 1. At the same stage of anaesthesia these reactions were observed in nine of the 18 patients of the saline group compared with only two of the 17 patients in the lignocaine group. Of these nine patients in the control group all had pupillary dilatation, two had lacrimation, and four coughed. In the lignocaine group only one patient responded to intubation by coughing.

Figure 2 displays the intraocular pressure in the control and lignocaine groups during induction of anaesthesia and immediately after the tracheal intubation.

The mean rise of the intraocular pressure of both groups reached its peak value during the first minute

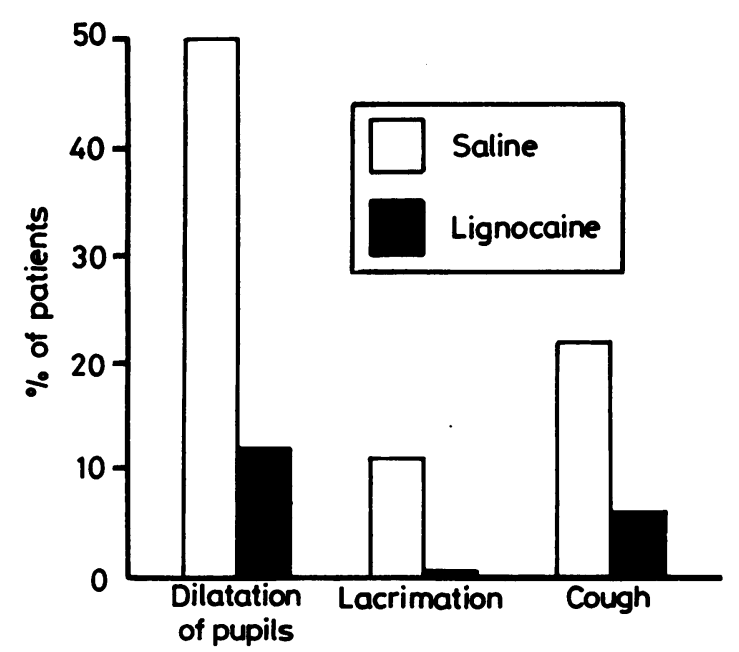

Fig. 1 Comparison of the ocular and systemic reactions to tracheal intubation in the saline versus lignocaine groups. after intubation and was $18.0 \mathrm{mmHg}$, SEM 0.9 , in the control group, and $14.9 \mathrm{mmHg}$, SEM 0.5 , in the lignocaine group. Initial values were $12.9 \mathrm{mmHg}$, SEM $0 \cdot 6$, and $14.2 \mathrm{mmHg}$, SEM $0 \cdot 5$, respectively (difference NS). The rise at 1 minute after intubation in the saline group was statistically significant compared with its initial recording $(<0.005)$; however, this was not the case in the lignocaine group. The difference in peak intraocular pressure between the two groups after tracheal intubation was also statistically significant $(p<0 \cdot 005)$.

Analysis of the intraocular pressures, of those patients who did not cough in the two groups showed essentially the same significant difference in pressure as shown in Fig. 2.

\section{Discussion}

During induction of anaesthesia the phase of tracheal intubation generally causes intense autonomic reflex

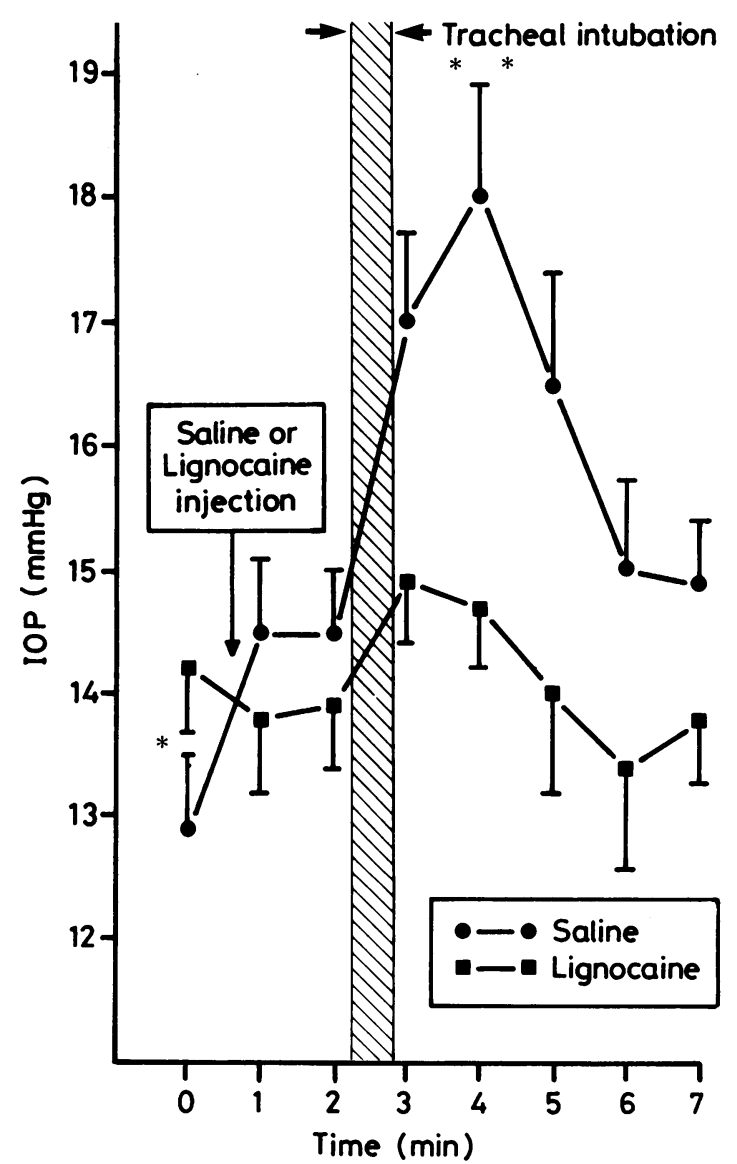

Fig. 2 Comparison of the intraocular pressure in experimental and control groups. ${ }^{*} p=N S .{ }^{* *} p=<0.005$. 
responses such as tachycardia, hypertension, and a rise in the intraocular pressure. ${ }^{1+6}$ These changes, which are short lived, probably have little influence on normal healthy individuals. However, in patients suffering from a lacerated globe ${ }^{56}$ even a mild rise in IOP as a result of these autonomic responses may be dangerous, especially when rapid sequence induction of anaesthesia has to be conducted.

The mitigation of IOP responses as presented in this study, with a rise of $5 \cdot 1 \mathrm{mmHg}$ in the control group compared with $0.7 \mathrm{mmHg}$ in the lignocaine group, may have an important implication in patients with penetrating eye injury. Attenuation of IOP responses by means of other anaesthetic agents such as fentanyl requires the administration of very high doses, which are not suitable for urgent cases and result in prolonged respiratory depression and delay in tracheal extubation.

A Schiøtz tonometer was employed owing to its ease of use and our need for relative and not absolute values of IOP. In spite of its disadvantages compared with applanation tonometry, the relative inaccuracy of the Schiøtz tonometer can be ignored in the healthy eye with average scleral rigidity and normal IOP. ${ }^{x 4}$

Among the factors affecting the intraocular pressure, changes in the systemic arterial blood pressure have the lowest significance owing to displacement of aqueous from the anterior chamber, which is equilibrated by a shift of blood from the choroidal vessels. ${ }^{611}$ A direct and immediate relation exists between the central venous pressure and intraocular pressure, and the rise in central venous pressure caused by coughing is instantly transmitted to the eye by impeding the efflux of aqueous. ${ }^{\circ}$ Laryngoscopy and intubation, as shown in this study, also have an important role in raising intraocular pressure, possibly as a result of the sympathetically mediated vasoconstriction generating increased venous return and a sudden rise in central venous pressure.' This suppression of ocular and cough reflexes is produced by the inhibition of polysynaptic spinal reflexes by lignocaine."

The observation of the described ocular and layrngeal reactions during induction of anaesthesia, especially as IOP is not routinely measured, will serve to indicate and alert the ophthalmologist to a possible rise in IOP during that period.
Children have brisk cardiovascular responses; they also have a higher incidence of perforating eye injury than adults. The use of intravenous lignocaine is especially indicated in this group of patients.

In strabismus surgery, a temporary decrease in IOP is observed due to manipulation of the globe during the operation, without any relation to the type of anaesthesia or to the type of muscle operated upon. ${ }^{12}$

We suggest that lignocaine exerts its effect on reducing intraocular pressure by mitigating autonomic reactions. Its administration before tracheal intubation in children suffering from lacerated eye is recommended, particularly when rapid sequence induction is needed in the anaesthesia of urgent ophthalmic operations.

\section{References}

1 Hamill JF, Bedford RF, Weaver DC, Colohan AR. Lidocaine before endotracheal intubation: intravenous or laryngotracheal? Anesthesiology 1981; 55: 578-81

2 Abou-Madi MN, Keszler H, Jacoub JM. Cardiovascular reactions to laryngoscopy and tracheal intubation following small and large intravenous doses of lidocaine. Can Anaesth Soc J 1977: 24: 12-9.

3 Stcinhaus JE, Gaskin L. A study of lidocaine as a suppressant of cough reflex. Anesthesiology 1963; 24: 285-90.

4 Meyers E, Krupin T, Johnson M, Zink H. Failure of nondepolarizing neuromuscular blockers to inhibit succinylcholineinduced increased intraocular pressure. A controlled study. Anesthesiology 1978; 48: 149-51.

5 Joshi C, Bruce DL. Thiopental and succinylcholine: action on intraocular pressure. Anesth Analg 1975; 54: 471-5.

6 Duncalf D, Foldes FF. Effect of anesthetic drugs and muscle relaxants on intraocular pressure. In: Smith RB, ed. Anesthesia in ophthalmology. Int Ophthalmol Clin 1973; 13: 21-34.

7 Drenger B, Pe'er J, BenEzra D, Katzenelson R, Davidson JT. The effect of intravenous lidocaine on the increase in intraocular pressure induced by tracheal intubation. Anesth Analg 1985; 64: $1211-3$

8 Kaufman HE. Pressure measurement: which tonometer? Invest Ophthalmol Vis Sci 1972; 11 : 80-5.

9 Drance SM. The coefficient of scleral rigidity in normal and glaucomatous eyes. Arch Ophthalmol 1960; 63: 668-74.

10 Kaskel D, Baumgart W, Metzler U, Fink H. Blood pressure, blood flow and intraocular pressure. Ophthalmic Res 1974; 6: 338-45.

11 De Jong RH. Physiology and pharmacology of local anesthesia. Springficld: Thomas, 1970: 144-57.

12 Pc'er J, Drenger B, BenEzra D. Intraocular pressure variations during strabismus surgery. J Pediatr Ophthalmol Strabismus 1986; 23: 98-1(0).

Accepted for publication 2 September 1986. 